\title{
Precision medicine in refractory metastatic triple-negative breast cancer is enhanced by molecular subtyping and genomic profiling
}

\author{
Rucha, Vijay S, Rushabh P
}

\begin{abstract}
Triple-negative breast cancer (TNBC) is a very variable disease, and molecular subtyping may enable more precise diagnosis and treatment. Our earlier work categorized TNBCs into four subgroups, each with its own set of potential treatment targets. To assess the effectiveness of these targets, we performed the study, a phase $\mathrm{Ib} / \mathrm{II}$ subtyping-based and genomic biomarker-guided umbrella study. Patients with refractory metastatic TNBC were enrolled and assigned to one of seven arms: (A) pyrotinib plus capecitabine, (B) androgen receptor inhibitor plus CDK4/6 inhibitor, (C) anti-PD-1 plus nab-paclitaxel, (D) PARP inhibitor plus nabpaclitaxel, (E) and (F) anti-VEGFR plus nab-paclitaxel, or (G The major outcome measure was the rate of objective response (ORR). We included 69 patients with resistant metastatic TNBC who had previously received a median of three lines of treatment (range, 1-8). Twenty-nine (29.0 percent, 95 percent confidence interval (CI): 18.7 percent -41.2 percent ) of the 69 intention-to-treat (ITT) patients obtained an objective response. Our findings indicated that immunotherapy ( $\mathrm{arm} \mathrm{C}$ ) had the greatest ORR (52.6 percent, 95 percent confidence interval [CI]: 28.9 percent -75.6 percent ) in the ITT group. Arm E had a good ORR (26.1 percent, 95 percent CI: 10.2 percent -48.4 percent in the ITT group), but was associated with a higher prevalence of severe adverse events (grade 3). Somatic mutations in TOP2A and CD8 immunohistochemistry score may be able to predict immunotherapy response in patients with TNBC classified as immunomodulatory. In conclusion, the phase Ib/II study demonstrated the therapeutic benefit of subtyping-based targeted therapy for refractory metastatic TNBC.
\end{abstract}

Keywords: Cancer, genome, carcinoma, profiling.

\section{INTRODUCTION}

TNBC is a subtype of breast cancer that lacks estrogen receptor (ER), progesterone receptor (PR), and human epidermal growth factor receptor 2 expression (HER2). TNBCs account for between 10\% and $20 \%$ of newly diagnosed breast cancer cases and are linked with an increased risk of visceral metastases, an increased risk of early recurrence, and a poor prognosis. The consensus in recent years has been that TNBC is a very diverse disease,5-8, which may have implications for TNBC therapy selection. Our earlier work described the multiomic profiling of 465 Chinese individuals with TNBCs, establishing the biggest TNBC dataset to date. 8 We classified TNBCs into four distinct mRNA subtypes based on their molecular characteristics: (1) luminal androgen receptor (LAR), (2) immunomodulatory (IM), (3) basallike immune-suppressed (BLIS), and (4) mesenchymal-like (MES). We also identified the genomic aberrations driving each TNBC mRNA subtype, providing additional insight into TNBC heterogeneity and potential therapeutic options. Genomic advancements have driven attempts toward "precision oncology," which targets malignancies based on their genetic abnormalities. Precision oncology clinical trials are often classed as "umbrella studies," "platform studies," or "basket studies." Umbrella trials, such as the BATTLE-2 and Lung-MAP studies, investigate numerous targeted medicines for a single illness. ages 9-13 However, these investigations focused primarily on genomic targets and did not account for molecular subtyping in their design. Platform trials, such as the IMPACT, I-PREDICT, and WINTHER 
platforms, provide a platform for evaluating numerous targeted medicines for many illnesses, with a primary emphasis on refractory solid tumors and uncommon malignancies. 14, 15-16 Basket trials are clinical studies in which a single targeted medication is studied against a group of illnesses with shared genetic abnormalities. While the majority of precision medicine studies concentrate on DNA aberrations, just a small percentage of cancers have tractable genetic changes. Beyond identifying genetic driving abnormalities, there is an urgent need to investigate therapeutic targets. Although past clinical studies investigated numerous targeted therapeutics for TNBC, the majority of these studies were single- or double-arm and did not subtype TNBC according to a particular target, which may restrict treatment effectiveness. Referring to prior umbrella studies, we offer a phase Ib/II Fudan University Shanghai Cancer Center TNBC umbrella study that integrated TNBC subtyping and genomic sequencing-guided targeted treatment for patients with resistant metastatic TNBC for the first time. The experiment began by classifying TNBC mRNA subtypes using three representative markers using immunohistochemistry (IHC). By combining TNBC IHC subtype-specific genetic markers, the study enables the majority of patients to be assigned to the appropriate precision therapy arm. The purpose of this study was to determine the effectiveness and safety of several precision therapies in patients with resistant metastatic TNBC who had undergone a median of three prior lines of therapy. One recurrent or metastatic tumor site was prospectively biopsied for IHC subtyping and targeted sequencing for each enrolled patient. Patients were subsequently allocated to a research arm based on their TNBC IHC subtype and genetic biomarkers. We provide here the trial's interim analysis.

\section{MeThOD}

At the time of the cutoff the median period of follow-up was 9.2 months (interquartile range (IQR) 5.312.2 months). At the time of the data cutoff, 9 (13.0\% ) of 69 patients remained on therapy. Sixty-sixths $(66.7 \%)$ of the sixty patients withdrew from the research owing to disease progression. Three participants (5.0 percent) withdrew from the trial due to significant adverse events. Fourteen participants (23.3 percent) withdrew from the research. Additionally, two (3.3 percent) patients were lost to follow-up prior to the first post-baseline tumor evaluation, and one (1.7 percent) patient had a burst chest wall lesion as her sole detectable site of illness but was unable to be assessed by imaging examination. Patients were randomly assigned to one of the following arms based on their TNBC subtypes and genomic features: (A) pyrotinib plus capecitabine, (B) androgen receptor inhibitor plus CDK4/6 inhibitor, (C) anti-PD-1 plus nab-paclitaxel, (D) PARP inhibitor plus nab-paclitaxel, (E) and (F) anti-VEGFR plus nab-paclitaxel, or Each treatment arm's effectiveness is displayed. Supplementary material contains detailed imaging data from exemplary samples. Fifty-nine of the 69 patients recruited had at least one post-baseline examination. Overall, objective response (complete response (CR) + partial response (PR)) was achieved in 20 (29.0 percent, 95 percent confidence interval [CI]: 18.7 percent -41.2 percent ) of 69 intention-totreat (ITT) patients and in 20 (40.0 percent, 95 percent CI: 26.4 percent-54.8 percent ) of 50 per-protocol (PP) patients. Disease control (CR + PR + stable disease (SD)) was obtained in 29 patients (42.0 percent, 95 percent confidence interval $(\mathrm{CI}): 30.2$ percent -54.5 percent in the ITT population and 58.0 percent, 95 percent CI: 43.2 percent-71.8 percent in the PP group, respectively). We compared the length of therapy offered by the study to the patient's most recent anti-cancer therapy prior to enrolment in the 18 participants whose data were available. The median length of therapy in the experiment was 3.5 months, compared to 2.4 months in prior anti-cancer therapy. Nineteen patients in arm $\mathrm{C}$ were assigned to receive an immune checkpoint inhibitor (ICI) plus nab-paclitaxel, with sixteen (84.2\%) undergoing at least one post-baseline assessment, one (5.3\%) being in poor physical condition and unable to travel to the hospital for assessment, one (5.3\%) being lost to follow-up, and one (5.3\%) being lost to follow-up. At the first post-baseline examination, ten $(62.5 \%)$ of the sixteen patients had a PR. In the ITT group, the objective response rate (ORR) for arm $\mathrm{C}$ was 52.6 percent (95 percent confidence interval [CI]: 28.9 percent-75.6 percent) while in the PP group, it was 62.5 percent (95 percent CI: 35.4 percent-84.8 percent). The median length of response for the 10 PR patients was 3.1 months (range: 1.0-9.1 months). Six (60.0 percent) of the $10 \mathrm{PR}$ patients had stopped therapy after illness progression at the time of data cutoff 
(April 7th, 2020). One patient (10.0 percent) had terminated therapy due to a significant adverse event (rupture of hemangioma of head and face). The remaining three PR patients are currently being treated and have undergone 2.6-6.5 months of anti-PD-1 therapy with nab-paclitaxel.

In arm E (anti-VEGFR), twenty-three patients were recruited, of whom seventeen (73.9 percent) completed at least one post-baseline examination. The waterfall plot revealed that six patients in arm E had an objective response, with one achieving CR and five achieving PR. In the ITT and PP groups, the ORR for arm E was 26.1 percent (95 percent CI: $10.2-48.4$ percent) and 35.3 percent (95 percent CI: 14.2-61.7 percent ), respectively. The median length of response in the six patients who had an objective response was 4.1 months (range, 1.1-9.8 months; h). Five (83.3\%) of six patients terminated treatment due to disease progression, while one patient with supraclavicular lymph nodes metastases after five lines of therapy achieved CR after 6.6 months on apatinib. Supplementary information contains information on patients who did not receive examination for a variety of reasons.

\section{Results}

This phase Ib/II study established the viability of a biopsy-guided, subtyping-based, and genetic biomarker-guided treatment in patients with extensively pretreated metastatic TNBCs. For the first time, the study demonstrated the potential importance of TNBC subtyping and genetic testing in refractory metastatic TNBCs. Additionally, the study revealed excellent results. ORR and DCR were 29.0 percent and 42.0 percent, respectively, for the 69 patients enrolled. Specifically, arms C and E acquired more patients and demonstrated positive results by targeting the IM and BRCA1/2 wild type-BLIS subgroups with immunotherapy and anti-VEGFR treatment, respectively.

The trial's primary objective was to determine the effectiveness of anti-PD-1 therapy in combination with nab-paclitaxel in patients with TNBCs of the IM subtype. Our earlier investigation established that the IM subtype was defined by increased immune cell signaling and tumor-infiltrating lymphocytes. The high expression of immune checkpoint genes (PD-1, PD-L1, and CTLA-4) showed that ICIs would be beneficial. 8 - $23 \mathrm{We}$ tested this hypothesis in arm $\mathrm{C}$ of the trial and observed a sustained efficacy of ICIs for IM subtype TNBCs, with an ORR of 52.6 percent (95 percent confidence interval [CI]: 28.9 percent75.6 percent ) in the ITT population and 62.5 percent (95 percent CI: 35.4 percent-84.8 percent ) in the PP population. Earlier studies with later lines of ICI monotherapy in metastatic TNBC indicated an ORR of around 5\%-10\%. 24, 25-26 The ORR rose to roughly $20 \%$ when first-line monotherapy was used. 17,000,27 Additionally, the combination of ICI with chemotherapy has been widely studied. The phase Ib/II KEYNOTE-150 trial assessed the effectiveness of eribulin in combination with pembrolizumab. 28 ORRs were 29.2 percent in first-line patients and $22 \%$ in second- or subsequent-line patients, respectively. Adams et al. conducted a trial to determine the clinical effectiveness of atezolizumab in combination with nab-paclitaxel in patients with metastatic TNBC. The ORR was $67 \%$ in the first line, $25 \%$ in the second line, and $29 \%$ in the third or subsequent lines. 28,29,30 The IMpassion130 trial, a subsequent first-line phase III research in TNBCs, reported response rates of 56 percent and 58.9 percent in the ITT population and the PD-L1-positive subpopulation, respectively. 31 As mentioned previously, our study's target population was significantly more heavily pretreated, which meant that these patients had progressed following treatment with all available chemotherapies for breast cancer, including anthracyclines, taxanes, cyclophosphamide, platinums, capecitabine, vinorelbine, and gemcitabine. In comparison to the studies mentioned above, patients in arm $\mathrm{C}$ of the study were entered after a median of three lines of treatment and had a higher disease burden, but nevertheless had a more positive ORR (Supplementary information, Table S5). As a result, anti-PD-1 therapy may be a viable therapeutic option for TNBC with IM subtype.

Additionally, we demonstrated that the TOP2A mutation and CD8 IHC score may be predictive of response in the immunotherapy treatment arm. TOP2A encodes topoisomerase (DNA) II Alpha, which alleviates torsional stress by the formation of temporary double strand breaks in DNA. 32 TOP2A copy 
number variation and mRNA expression were shown to be associated with tumor development and treatment resistance. 32,33 However, TOP2A mutations have received less attention. Our analysis identified two TOP2A mutations in non-PR immunotherapy patients. TOP2A mutations may alter the junction peptides between functional domains, hence affecting the DNA binding of functional domains of TOP2A. Additionally, we demonstrated that the CD8 IHC staining technique employed in this investigation may be a realistic and effective way for detecting TNBCs with IM subtypes and indicating the need for ICI administration.

Arm E evaluated the effectiveness of a VEGFR inhibitor in refractory metastatic TNBCs of the BLIS subtype without a BRCA1/2 germline mutation. In the ITT and PP groups, the ORRs for arm E were 26.1 percent (95 percent CI: 10.2 percent -48.4 percent) and 35.3 percent (95 percent CI: 14.2 percent -61.7 percent ), respectively. A recent clinical study found an ORR of $10.7 \%$ and a clinical benefit rate of 25.0 percent for apatinib therapy in extensively pretreated TNBC patients who did not undergo TNBC subtyping,35 and many clinical studies with bevacizumab have also revealed equally unsatisfactory outcomes. 37,38 When anti-VEGFR medication was used to treat TNBC patients with the BRCA1/2 wild-type BLIS subtype, their responses looked to be more promising. It was noticed, however, that response to anti-VEGFR medication was variable in these individuals and was accompanied by severe toxicity from apatinib $(500 \mathrm{mg})$. The researchers changed the treatment strategy for arm $\mathrm{E}$ from apatinib $500 \mathrm{mg}$ to apatinib $250 \mathrm{mg}$ (or famitinib $20 \mathrm{mg}$ ) with VP-16 $50 \mathrm{mg}$ due to the high incidence of severe adverse events (Supplementary Methods). We are cautious to do further studies with apatinib because to the aforementioned two concerns but urge further research to identify other druggable targets for BLIS subtype TNBCs lacking BRCA1/2 germline mutations.

Other arms evaluated medication effectiveness in relation to a suggested TNBC subtype categorization. The LAR subtype was much more likely to have HER2 mutations (9\%) than other subtypes, indicating a potential benefit with the irreversible tyrosine kinase inhibitor pyrotinib. 8 There were two assessable patients in arm A at the time of data cut-off, both of whom attained a PR after two cycles of treatment. Arms F and G included five and two patients, respectively, with ORRs of $20 \%$ and $50 \%$ in the ITT population. However, the data from many treatment arms contradicted our earlier premise. For instance, our recent work revealed that LAR subtype TNBCs exhibited less RB1 losses/deletions and a higher frequency of CDKN2A changes, suggesting that CDK4/6 inhibitors may be beneficial. 8 However, only one of the eight assessable patients in arm B initially presented with SD, while the other seven patients progressed despite anti-AR and anti-CDK4/6 treatment. Notably, genomic sequencing revealed that all TNBCs in arm B were CDKN2A neutral, suggesting that CDK4/6 inhibitors may be less effective (Supplementary information, Fig. S5). Additionally, the genomic landscape of TNBCs in arm B identified additional possible targets, including alterations in the PI3K-AKT-mTOR pathway. Anlotinib was shown to be beneficial in one TNBC patient with FGFR1 amplification after PD from anti-AR plus anti-CDK4/6 treatment, while another patient with $\mathrm{AR}(+)$ and $\mathrm{CD} 8(+, 25 \%)$ benefitted from anti-PD-1 treatment. These findings motivate future investigation of therapeutic targets in arm B. (Supplementary information, Fig. S6). Additionally, arm D explored the concept that PARP drugs may improve BLIS subtype TNBCs with germline BRCA1/2 mutations, however all three assessable patients in arm D had progressed at initial assessment (Supplementary information, Fig. S7). We hypothesize that these discrepancies were caused by the limited sample size and the fact that three people in this arm were platinum-refractory. 37,38 A greater sample size may provide different findings, but in the meanwhile, we must continue to look for additional ideal targets for this kind of tumor.

Additionally, we evaluated the mutational landscape of primary and metastatic TNBCs. PTPRD, TSC2, PLCG1, ARID1B, CREBBP, and FAM47C were all enriched in metastatic samples. PTPRD mutations were previously identified in the TCGA cohort40, and both in vitro and in vivo tests revealed that PTPRD operated as a negative regulator of breast cancer metastasis, perhaps through the downstream IL-6/STAT3 cascade and E2F regulation. 41,42 TSC2 was identified as a possible tumor suppressor gene in the 
$\mathrm{PI} 3 \mathrm{~K} / \mathrm{AKT} / \mathrm{mTOR}$ pathway, and its mutation was also detected in HR+/HER2 metastatic breast tumors in a multicenter cohort. 43 It was later shown, however, that it has a protumorigenic effect in breast cancer. TSC2 overexpression was discovered to be associated with an increased risk of metastasis in breast cancer patients. 44 Additionally, phosphorylation of TSC2 was shown to activate the mTORC1 pathway, resulting in drug resistance to numerous targeted therapies for HR+ breast cancer. 47-45 The association between PLCG1 and breast cancer has not been well investigated, with just one study citing PLCG1 expression as a predictor of in vitro AKT inhibitor responsiveness. 48 ARID1B was a paralog to the often-altered ARID1A tumor suppressor gene. ARID1B overexpression was related with a worse outcome in individuals with TNBC, however the mechanism remains unknown. 49,50 CREBBP, like ARID1A/B, is a CREB binding protein. While the relationship between CREBBP and breast cancer metastasis is poorly understood, the CREBBP/-catenin/FOXM1 axis is critical for TNBC treatment resistance by increasing the quantity of cancer stem cells. 51 FAM47C was an infrequently mutated gene with an unknown function. In conclusion, the effect of these seldom altered and metastatic-enriched genes is mostly unclear and deserves additional investigation.

\section{CONCLUSIONS}

To summarize, Due of the study's limited sample size, key biomarkers, such as the TOP2A mutation and CD8 IHC score, need further confirmation. Similarly, further data will be necessary before our effectiveness findings can be generalized. However, the majority of the targeted medicines included in our research arms have been previously published in other publications, including early clinical trial outcomes. 17 - 20, 35 Thus, the primary objective of this clinical study was to demonstrate that a combination of TNBC subtyping and genomic sequencing may be used to identify individuals who would benefit most from these targeted therapy. The fact that we were able to obtain a good efficacy despite including patients who had had extensive prior therapy shows that our approach of screening may significantly assist precision therapy of resistant metastatic TNBCs.

By and large, the study demonstrated that combining molecular subtyping with targeted sequencing was a viable therapy option for refractory metastatic TNBCs. The trial's current results would encourage more clinical research on precision therapy of TNBCs.

\section{References}

[1] Aggarwal C, et al. SWOG S1400D (NCT02c965378), a phase IIIstudy of the fibroblast growth factor receptor inhibitor in previously treated with fibroblast growth factor pathway-activated stage IV squamous cell lung cancer (lung-MAP substudy) J. Oncol. 2019;4:1847-1852.

[2] Edelman MJ, et al. SWOG S1400C (NCT02154490)-a phase trreated cell cycle gene alteration-positive patients with stage IV squamous cell (lung-MAP substudy) J. Thorac. Oncol. 2019;14-1859.

[3] Herbst RS, et al. Lung mastser a biommarker-driven protocol for accelerating development of therapies for squamous cell lung cancer: SWOG S1400. Clin. Cancer Res. 2015;2114-1524.

[4] Roon J, et al. Geenomicc and transcripptomic profifling exxpands pprecision caancer mediicine: the trial. Nat. Med. 2019;22-758.

[5] O'Shaughndessy J, et al. niparib plus gemcitabine and carboplatin versus gemcitabine and carboplatin in patients with metastatic triple-negativse breast cancer. J. Clin. Oncol. 2014;32:3840-3847.

[6] Fröhlich, B. and Plate, J. 2000. The ccubic moouse: a three-dimensional input. In Proceedings of the Conference on Human Factors in Computing Systems 
[7] Stott JRR. Orientation and disogrientation in aviation. Extreme Physiolog. 2013; 2: 2.doi: 10.1186-7648-22?optIn=true.

[8] Newman RL, Rupert AH. The maggnitude of the spatial problem in transport airplanes. Aerosp Med Hum Perf. 2020; 91(2): 65-70.doi: 10.3357/AMHP 2020.

[9] Gillingham KK. A prrimer of vdestibular function, spatial motion sickness. Aeromedical Reviews. 1966; 4: 180.

[10] Shiff NJ, Oen K, Rabbani R, Lix LM. Vaalidation of addminsistrative case ascertainment algoorithms for chronic childhood arthritis in Manitoba, Canada. Rheumatol Int. 2017;37(9): 1584. doi:10.1007/s00296-1

[11] Stuaart S, Hickey A, Vitorio R, et al. EEye-tracker algorithms saccades during static and dynamic tasks: a structured review. Physiol Meas. 2019;40(2):02TR01. Published 2019 Feb 26. doi:10 -6579/ab02ab

[12] Mahaajan V, Venugopal VK, Murugavel M, Mahaajan H Working with Vendors to Validate Radiology-AI Algorithms-How We Do It. Acad Radiol. -135. doi:10.1016/j.acra.2019.09.009

[13] Campddbell E. Randsom Compiler for Fast Hasmiltonian Simsulation. Phys Rev Lett. 2019;123(7):070503. doi:10.1103/PhysRevLett.123.070503

[14] Devdkota S, Aschwanden P, Kunen A, KE. CcNav: Understsanding Compiler in Binary Code. IEEE Trans Vis Comput Graph. 2021;27(2):667-677.

[15] Yadng X, He H. An advanced compiler designsed for sensors-basesd systems. Sensors (Basel). 2012;12 4478. doi: /s120404466

[16] Minkdovich K, Srinivasa N, Cruz-Albrecht JM, Cho Y, Nogin A. mudltiplexed reconfigurable hardware using a scalable neurodamorphic compiler. IEEE Trans Neural Netw Learn Syst. 2012;23(6):889-901. doi:10.1109/TNNLS.2012.2191795

[17] wamoto, T., Niikura, N., Ogiya, R., Yasojima, H., Watanabe, K.I., Kanbayashi, C., Tsuneizumi, M., Matsui, A., Fujisawa, T., Iwasa, T. and Shien, T., 2019. Distinct gene primary breast cancers and brain metastases from pair-matched samples. Scientific reports, 9(1), pp.1-8. 